\title{
Maximum life span predictions using the Gompertz tumour Growth model
}

\author{
M. Pitchaimani ${ }^{1}$ and G.Somasundara Ori ${ }^{* 2}$ \\ 1,2 Ramanujan Institute for Advanced study in Mathematics, University of Madras, Chennai 600005, India.
}

\begin{abstract}
Studies in the evolutionary biology of cancer research require good estimates of the intrinsic growth rate of the tumour coefficient. A Gompertzian model is a classical continuous model useful in describing population dynamics; in particular, it is a very efficient mathematical modelto describe tumour growth in humans and animals. The Gompertz survival model of a tumour growth is the interest of many investigators in experimental biology and the evolutionary biology of ageing. Standard parameter estimation techniques, such as regression and maximum likelihood analysis, require knowledge of actual lifespan for parameter estimation to be successful. In this paper we introduce an alternative algorithm for estimating this parameter. And we examine maximum life span predictions through the Gompertz tumour growth model for large number of tumour cells at particular time.
\end{abstract}

Keywords: Asymptotic, Gompertz model, Maximum lifetime of tumour cells, Tumour doubling time. AMS classiffication code: $92 B 05,35 A 02$.

\section{Introduction}

Attempts to extend an individual's life beyond the onset of tumours is nothing new; in fact, surgeries to remove tumours were described in a work by physician Aetius of Amida (593 A.D.) [4]. There is now a great body of work dedicated towards understanding the development and growth of tumours.

As with most studies of population dynamics, mathematical modelling can provide great insight into the dynamics of tumour growth. The models that form the focus of this paper are tumour-level analyses (as opposed to cellular-level analyses) of avascular tumours. An avascular tumour is one that does not yet have blood vessels, and so the only method for the transportation of nutrients throughout the tumour is diffusion. As a result, at some point the cells in the centre of the tumour will not have enough nutrient to survive and will die off.

Given that the event, or mutation, that initially triggered the tumour probably occurred several years earlier, it is natural to ask why the tumour was not detected sooner and why it suddenly started to grow rapidly. Eventually this avascular tumour will reach an equilibrium size (2 $\mathrm{mm}$ in diameter, [15]), at which the rates of cell proliferation and apoptosis, averaged over the tumour volume, balance. At this stage the tumour typically comprises an outer rim of proliferating cells, a central core of necrotic debris and an intermediate region of quiescent cells which are alive, but do not proliferate due to nutrient deprivation [36, 37].

Possible theoretical bases of Gompertz tumour growth model have been addressed in the literature from various points of view, and it remains to be a topic of investigation [2,9,13,14,20,21, 25-30, 39]. Most of the authors have attempted to derive the Gompertz model as an approximation (or a special case) of more general models, which are deemed to be based on accepted biological foundations. A somewhat similar approach is pursued in this paper: the Gompertz model is postulated (based on its empirical justification) and then the more general model is specified to yield the Gompertz model.

Traditionally mathematical models describing solid tumour growth assume radial symmetry of the tumour and focus on its responses to various growth factors $[8,24,26]$. These models show excellent agreement with experimental results, reproducing the multi-layered structures that characterise solid tumours and multicellular spheroids. However, the deterministic Gompertz law of population growth has been widely used to describe in vivo tumour growth in experimental oncology $[5,6,16,33,34,38]$. The Gompertz law models the cells growth by the equation

$$
G(t)=A e^{-\beta t},
$$

where $\mathrm{A}$; the intrinsic growth rate of the tumour, is a parameter related to the initial mitosis rate and $\beta$; the growth deceleration factor. The corresponding Gompertz growth function can be obtained by integrating the growth rate function equation(1) of the following form

$$
V^{*}(t)=e^{\frac{A}{\beta}\left(1-e^{-\beta t}\right)}
$$


where $V^{*}(t)=\frac{V(t)}{V 0}$ and $V(t)$ is the clonogenic tumour volume at time $t ; V_{0}$ is the clonogen number at time $t=0: \quad A$ and $\beta(>0)$ are the Gompertz growth parameters. From a biological point of view, a greater $\beta$ value or a smaller $A$ value indicates a greater antitumoural effect of the therapy [11].

By setting $V^{*}(t)=\frac{V(t)}{V 0} \quad$ we obtain the approximation

$$
\mathrm{t}_{\mathrm{m}}=-\frac{1}{\beta} \ln \left[1-\frac{\beta}{\mathrm{A}} \ln \left(\mathrm{V}^{*}\left(\mathrm{t}_{\mathrm{m}}\right)\right)\right]
$$

We assume that $\mathrm{V}\left(\mathrm{t}_{\mathrm{m}}\right)$ maximum volume of tumour cells (where $\mathrm{t}_{\mathrm{m}}$ is the time at which the tumour contains a cell number which is one less than its maximum i.e., one cell less to death, and which approximates the maximum lifespan of tumour cells $\mathrm{t}_{\mathrm{m}}^{*}$ )

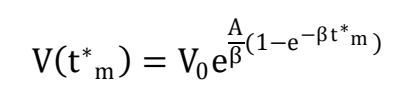

Equation (3) gives

$$
\ln \left[\mathbf{V}^{*}(t)\right]=\frac{A}{\beta}\left(1-e^{-\beta t}\right),
$$

Or

$$
\frac{A}{\beta}=\frac{\ln \left(V^{*}(t)\right)}{\left(1-e^{-\beta t}\right)}
$$

Where $\mathrm{V}^{*}(\mathrm{t})=\mathrm{V}(\mathrm{t}) / \mathrm{V}_{0}$

The cumulative intrinsic volume growth rate $V c$ of the Gompertz model of equation (2), is defined $V_{c}=$ $\int_{0}^{\infty} \mathrm{V}^{*}(\mathrm{t}) \mathrm{dtSubstitute}$ the value of $\mathrm{V}^{*}(\mathrm{t})$ from the equation (2) in the above equation and apply a little algebra we get the following equation

$$
-\beta=\frac{1}{V_{c}} e^{-\frac{A}{\beta}} \int_{-\frac{A}{\beta}}^{\infty} \frac{e^{-z}}{z} d z
$$

Where $z=-\frac{A}{\beta} e^{-\beta t}$. Clearly, the above integral (6), exists $\forall \beta \in R$.

Consider the initial volume of size $\mathrm{V}_{0}$ at $\mathrm{t}=0$. From the equation (1) the volume at which the growth rate of initial volume $V 0$ has increasing or, equivalently, it tends to a $V\left(t^{*}{ }_{m}\right)$ is the time at which volume approximates the maximum number of tumour cells, is called a critical time, $t_{\mathrm{k}}$ :

The remaining tumour cells from an original volume size $V(t)$, surviving at this critical time is called critical volume $V_{\mathrm{k}}$ and the corresponding Gompertz parameter in equation (1), is called critical Gompertz parameter $\beta_{\mathrm{k}}$.

The Gompertz model presents a doubling time (Volume Rate Doubling time (VRD)) which depends only on $\beta$. Comparisons of volume data of solid tumours in tumour growth model are aided by calculation of the VRD, because VRD changes in the same direction as lifespan of tumour cells.

The VRD changes with time Solving equation (2) for VRD gives

$$
\mathrm{VRD}=-\frac{1}{\beta} \ln \left[1-\frac{\beta}{\mathrm{A}} \ln (2)\right]
$$

Benjamin Gompertz (1825) [17] proposed that the growth of tumour volume increased exponentially with time for all tumours. Various subsequent researchers, especially in biology and gerontology, have viewed Gompertz observation as a law that describes the process of senescence in almost all type of tumours at any time after the onset of growth. As a rough approximation at initial growth, Gompertz exponential formula does capture the rise in growth in a great variety of tumours.

Until recently, it was impossible to determine whether this exponential rise continued for long period of time. For some tumours, the scattered data available suggested that growth rate decelerated at a long period of time, but questions about data reliability precluded strong conclusions. For other tumours, virtually nothing was known about growth rate at a very long period of time because the tumour cell growth studied had been too small to permit dependable estimates of growth at time that only a small fraction of the starting cohort reached.

It is well known that among most of the tumours, growth rate of volumes are generally lowest at initially and the accelerate at a constant rate during the major phase of middle lifespan.

When examined from initial onwards, at least up through the average lifespan. However, extensive deviations from the Gompertz model were recently documented, in which growth rate accelerations slow 
markedly by the average lifespan. After some time (critical time), the growth rate appears to cease increasing and may even decrease at these extremely long time. Decreasing growth rate at a long period of time can be predicted by the Gompertz law. In extreme time, growth rates may level off or even decline, after this stage how much the tumour volume can grow and when this biologically existing tumour will disintegrate, that time is the maximum lifespan of the tumour. We are going to estimate this maximum lifespan of the tumour $\mathrm{t}^{*} \mathrm{~m}$.

We wish to point out that our approach is not restricted to tumour growth only. The Gompertz model have been almost universally used to describe the growth of organisms, tissues and populations of single cell organisms. Additionally the biological assumptions and mathematical generality of the Gompertz model are sufficient to warrant its application to growth in general. The plan of this paper is as follows. In section 2 we find the behaviour of solid tumour growth Gompertz parameter and define critical volume Vk, critical growth time tk. Section 3 define the most important parameter maximum life span time $t^{*}{ }_{m}$. In section 4 we had discussion and conclusion.

\section{Estimating the upper limits of longevity}

Generally growth rates of the tumours in animals are low at initial time and then accelerate to a constant rate during the major phase of its life time. In equation (6) we estimated the Gompertz growth rate parameter $\beta$ and also we derived the equation for VRD to calculate how fast the initial volume will reach the maximum volume.

Before proceeding to find maximum lifespan we need to know the behaviour of the parameter and its sensitiveness with respect to volume, cumulative volume and time. This will give the results when the value of parameter $\beta$ increases/decreases accordingly the value of $\mathrm{t}_{\mathrm{m}}{ }_{\mathrm{m}}$ increases /decreases and estimate the value of critical Gompertz parameter.

\subsection{Behaviour of solid tumour growth Gompertz parameter}

Tofind the critical points of $\beta$ we consider the partials of $\beta$ with respect to $V^{*}(t), V_{c}$ and tm in equation (7). These are given by

And

$$
\begin{gathered}
\frac{\partial \beta}{\partial \mathrm{V}^{*}(\mathrm{t})}=\frac{\left[\mathrm{A}-\left(\frac{1}{V_{\mathrm{c}}}\right)\right] / \mathrm{V}^{*}(\mathrm{t}) \ln \mathrm{V}^{*}(\mathrm{t})}{1+\left(\frac{\mathrm{e}^{-\beta \mathrm{t}_{\mathrm{m}}}}{\left(\mathrm{e}^{-\beta \mathrm{t}_{\mathrm{m}}}-1\right)}\right) \mathrm{t}_{\mathrm{m}}\left[\mathrm{A}-\left(\frac{1}{\mathrm{~V}_{\mathrm{c}}}\right)\right]} \\
\frac{\partial \beta}{\partial \mathrm{V}_{\mathrm{c}}}=\frac{-\beta / \mathrm{V}_{\mathrm{c}}}{1+\left(\frac{\mathrm{e}^{-\beta \mathrm{t}_{\mathrm{m}}}}{\left(\mathrm{e}^{-\beta \mathrm{t}_{\mathrm{m}}}-1\right)}\right) \mathrm{t}_{\mathrm{m}}\left[\mathrm{A}-\left(\frac{1}{\mathrm{~V}_{\mathrm{c}}}\right)\right]}
\end{gathered}
$$

$$
\frac{\partial \beta}{\partial \mathrm{t}_{\mathrm{m}}}=\frac{-\beta\left(\frac{\mathrm{e}^{-\beta \mathrm{t}_{\mathrm{m}}}}{\left(\mathrm{e}^{\left.-\beta \mathrm{t}_{\mathrm{m}}-1\right)}\right) /\left[\left(\frac{1}{\mathrm{~V}_{\mathrm{c}}}\right)-\mathrm{A}\right]}\right.}{1+\left(\frac{\mathrm{e}^{-\beta \mathrm{t}_{\mathrm{m}}}}{\left(\mathrm{e}^{\left.-\beta \mathrm{t}_{\mathrm{m}}-1\right)}\right)}\right) \mathrm{t}_{\mathrm{m}}\left[\mathrm{A}-\left(\frac{1}{\mathrm{~V}_{\mathrm{c}}}\right)\right]}
$$

Here, $\beta \mathrm{tm} \geq 1$ and $[\mathrm{A} \leq(1 / \mathrm{Vc})]$ is positive, since

Therefore, for $\beta \mathrm{tm} \geq 1$ the value of

$$
\left(\frac{1}{V_{c}}\right) \leq A
$$

$1+\left(\frac{e^{-\beta t_{m}}}{\left(e^{-\beta t_{m}}-1\right)}\right) t_{m}\left[A-\left(\frac{1}{V_{c}}\right)\right]$ is positive.

This will give the result

$\frac{\partial \beta}{\partial \mathrm{V}^{*}(\mathrm{t})} \geq 0, \frac{\partial \beta}{\partial \mathrm{V}_{\mathrm{c}}} \leq 0$ and $\frac{\partial \beta}{\partial \mathrm{t}_{\mathrm{m}}} \leq 0$.

It shows that the value of parameter $\beta$ is increases when value of $\mathrm{V} *(\mathrm{t})$ is increases, $\beta$ is decrease when the value of $\mathrm{Vc}$ increase and also $\beta$ is decrease when the value of tm increases. If we send $V^{*}(t)$ to $\infty$ in (8),(9) and (10) get that

Also we obtain,

$$
\lim _{V^{*}(t) \rightarrow \infty} \frac{\partial \beta}{\partial V^{*}(t)}=0, \lim _{V^{*}(t) \rightarrow \infty} \frac{\partial \beta}{\partial V_{c}}=0 \text { and } \lim _{V^{*}(t) \rightarrow \infty} \frac{\partial \beta}{\partial t_{m}}=0 .
$$

$$
\begin{gathered}
\frac{\partial \beta}{\partial \mathrm{V}^{*}(\mathrm{t})}=0 \Leftrightarrow \mathrm{A}=\frac{1}{\mathrm{~V}_{\mathrm{c}}}, \quad \forall \mathrm{V}^{*}(\mathrm{t}), \\
\frac{\partial \beta}{\partial \mathrm{V}_{\mathrm{c}}}=0 \Leftrightarrow \beta=0,
\end{gathered}
$$




$$
\frac{\partial \beta}{\partial \mathrm{t}_{\mathrm{m}}}=0 \Leftrightarrow \mathrm{A}=\frac{1}{\mathrm{~V}_{\mathrm{c}}} \text { or } \beta=0 \text { or } \beta=-\infty .
$$

Upon substitution $A=\frac{1}{v_{c}} \quad$ in (4), we get

$$
\frac{t_{m}}{V^{*}(t) \ln V^{*}(t)}=\frac{\beta t_{m}}{\left(1-e^{-\beta t}\right)}
$$

from (11) it follows that,

$$
\frac{t_{m}}{V^{*}(t) \ln V^{*}(t)}\left\{\begin{array}{l}
<1 \text { if } \beta<0 \\
=1 \text { if } \beta=0 \\
>1 \text { if } \beta>0
\end{array}\right\}
$$

The

$\frac{t_{m}}{V_{c} \ln V^{*}(t)}=1$ at which $\beta$ changes sign is said to be the critical point $\beta \mathrm{k}$.

point

From [35], Collins and his co-workers [7] were able to show that for a series of 206 children with Wilms' tumour that risk of recurrence agreed well with theoretical prediction by the method of Boag [10] and also the growth rate function approaches a constant with predictions on the basis of exponential growth at larger time [1, $3,12,18,19,22,31,32]$.

To determine the critical Gompertz parameter, $\beta \mathrm{k}$, first we use the identity $\frac{t_{m}}{V_{c} \ln V^{*}(t)}=1$ to obtain critical values of $\beta$; namely critical volume, $V k$ and critical time tk. Since the partials of $\beta$ with respect to $V^{*}(t), \frac{1}{V_{c}}$ and $t_{m}$ become zero at $\frac{t_{m}}{V_{c} \ln V^{*}(t)}=1$.

Note that the condition $\frac{t_{m}}{V_{c} \ln V^{*}(t)}=1$ is necessary to have a constant growth rate function. Finally we obtain the asymptotic solution of (7), for the critical values, Vk and tk. Now we shall prove the existence of critical volume and critical time.

\subsection{Critical volume Vk}

For a given $V^{*}(t), \frac{1}{V_{c}}$ and $t_{m}$ with $\frac{t_{m}}{V_{c} \ln V^{*}(t)}>1$ there exists a critical volume $V k$ and is given by $\mathrm{e}^{\frac{t_{m}}{V_{c}}}$. Indeed, since $\ln V^{*}(t)<\frac{t_{m}}{V_{c}}$ we can take $\ln V^{*}(t)=\frac{t_{m}}{V_{c}}$, or $V_{k}=e^{\frac{t_{m}}{V_{c}}}$. For instance,(see TableI in [31] when (Mouse Krebs)) $\mathrm{A}=5: 25, \mathrm{tm}=15 ; 20$ and 25, we find that $\mathrm{Vk}=927_{\mathrm{x}} 10^{6} 6 ; 949_{\mathrm{x}} 10^{6}$ and $952 \times 10^{6}$, respectively. Note that $\mathrm{Vk}$ is increases as tm. Thus the remaining volume (critical volume) approximates $949 \mathrm{x}$ $10^{6}$ to $952 \times 10^{6}$ cells. Since the volume reaches its maximum size, the above said tm can be treated as critical time tk. From this we obtained tk $=\mathrm{Vc} \ln [\mathrm{Vk}]$. To study the tumor growth rate of the remaining critical volume we need to consider critical Gompertz parameter $\beta \mathrm{k}$ because $\frac{t_{m}}{V_{c} \ln V^{*}(t)}=1$ when $V^{*}(t)=V_{k}$. Thus, we conclude that when $\frac{t_{m}}{V_{c} \ln V^{*}(t)}>1, t k=t m$ and $V_{k}=e^{\frac{t_{m}}{V_{c}}}$. Clearly, when $\frac{t_{m}}{V_{c} \ln V^{*}(t)}>1$, both tk and tm are same.

\subsection{Critical growth time tk}

On the contrary, when $\frac{t_{m}}{V_{c} \ln V^{*}(t)}<1$ it is trivial to find the critical volume. As tm $<\mathrm{Vc} \ln [\mathrm{Vk}]$ we can take tk= $\mathrm{Vc} \ln [\mathrm{Vk}]$. For instance,(see Table I in [31] when (Rat R39 Sarcoma,R3a7)) $\mathrm{A}=1.28, \mathrm{tm}=42.44$ days and $\mathrm{V}^{*}(\mathrm{t})=241 \mathrm{~cm} 3$, we find that $\mathrm{tk}=28.4854$. Thus we conclude that when $\frac{\mathrm{t}_{\mathrm{m}}}{\mathrm{V}_{\mathrm{c}} \ln \mathrm{V}^{*}(\mathrm{t})}<1$, tk $=\mathrm{Vc} \ln [\mathrm{Vk}]$, $V_{k}=e^{\frac{t_{m}}{V_{c}}}$ and $t_{k} \neq t_{m}$.Clearly, when $\frac{t_{m}}{V_{c} \ln V^{*}(t)}<1, t k<t m$. Thus, in general, for any given $\frac{1}{V_{c}}$, tm and $V^{*}(t)$, we get $t_{k} \leq t_{m}$.

In the above subsections we checked the existence of critical Gompertz parameters Vk and tk. After the critical time the growth rate of tumour will starts diminish and will have more inuence on the growth of the tumour. So we needs to know the how much time the volume of tumour can exist and expand biologically as well as theoretically. This paper main aim to _nd that biological existence of tumour and that maximum lifespan time $\mathrm{t}_{\mathrm{m}}^{*}$.

\section{Maximal Life Span Time $\mathbf{t}_{\mathrm{m}}$}

If the critical volume $V_{k}$ is not arrived at $t_{m}$, then the value of tm can be taken as critical life Span $t_{k}$. Thus, the actual $t_{m}$ the time at which the critical volume has reached maximum lifespan is to be determined. First, for given $\frac{1}{V_{c}}, t_{m}$ and $V(t)$ with $\frac{t_{m}}{V_{c} \ln V^{*}(t)}=1$; the only solution of equation(5) and (6) is $A=\frac{1}{V_{c}}$, and $\beta=$ 
0 ; that is mortality rate remains constant. Then next, for a given $\frac{1}{\mathrm{~V}_{\mathrm{c}}}$, $\mathrm{t}_{\mathrm{m}}$ and $\mathrm{V}(\mathrm{t})$ with $\frac{\mathrm{t}_{\mathrm{m}}}{\mathrm{V}_{\mathrm{c}} \ln \mathrm{V}^{*}(\mathrm{t})}<1$; we get $\beta>0$ from (12). Thus, the growth rate becomes

$$
G(t)=\left\{\begin{array}{c}
\frac{1}{V_{c}}, \text { if } V(t)=V_{k} \\
A_{k} e^{\beta t}, \text { if } V(t)>V_{k}
\end{array}\right\}
$$

where $A_{k}$ the initial growth rate at critical age $t_{k}$, is given by (see equation (5))

$$
\frac{A_{k}}{\beta}=\frac{\ln \left(V^{*}(t)\right)}{\left(1-e^{-\beta t}\right)},
$$

To determine the $t_{m}$ consider the value of $V_{c}=\int_{0}^{\infty} V^{*}(t) d t$. We have

$$
V_{c}=\int_{0}^{t_{k}} e^{\frac{A}{\beta}\left(1-e^{-\beta t}\right)} d t+\int_{t_{k}}^{t^{*}} e^{\frac{A_{k}}{\beta}\left(1-e^{-\beta t}\right)} d t \equiv I_{\beta}+I_{-\beta}
$$

where $\mathrm{I}_{\beta}$ and $\mathrm{I}_{-\beta}$ denote the first and second integral respectively on the right hand side of equation (14). We note that $I_{-\beta}$ is the mean life length of $\mathrm{Vk}$ from $t_{k}$. To find the mean life length,we rewrite the equation(14) thus

$$
I_{-\beta}=V_{c}-I_{\beta}=\int_{t_{k}}^{\infty} e^{\frac{\ln \left(v^{*}(t)\right)}{\left(1-e^{\left.-\beta_{\text {asy }} t_{k}\right)}\left(1-e^{-\beta_{\text {asy }} t}\right)\right.}} d t
$$

here we have substituted asymptotic solution of (6) into $\mathrm{I}_{\beta}$ and it is given by (M.Pitchaimani and somasundara ori)

$$
\beta_{\text {asy }}=\left\{\begin{array}{ll}
-\frac{1}{t_{m}} \ln \left[1-e^{\left(\frac{\mathrm{t}_{m}}{V_{c}}\right) c /\left(\frac{\mathrm{t}_{m}}{V_{c}}-1\right)}\left(\ln V^{*}(t)^{\left(\frac{t_{m}}{V_{c}}\right) /\left(\frac{t_{m}}{V_{c}}-1\right)}\right)\right] & \text { if } \frac{t_{k}}{V_{c} \ln V^{*}(t)}<1 \\
-\frac{1}{t_{m}} \ln \left[1-\frac{C \ln \left[V^{*}(t)\right]-\ln \left[V^{*}(t)\right]-1}{\ln \left(\ln \left[V^{*}(t)\right]\right)+C}\right], & \text { if } \frac{t_{k}}{V_{c} \ln V^{*}(t)}=1
\end{array}\right\}
$$

where $\mathrm{C}=0: 577215$, Euler's constant.

A simple substitution in the integral (15) gives

$$
\begin{aligned}
& \mathrm{I}_{-\beta}=\frac{-1}{\beta_{\text {asy }}} \mathrm{e}^{\frac{\ln \left(\mathrm{V}^{*}(\mathrm{t})\right)}{\left(1-\mathrm{e}^{\left.-\beta_{\text {asy }} \mathrm{t}_{\mathrm{k}}\right)}\right.}} \int_{\frac{\ln \left(\mathrm{V}^{*}(\mathrm{t})\right)}{\left(1-\mathrm{e}^{\left.-\beta_{\text {asy }} \mathrm{t}_{\mathrm{k}}\right)}\right.}}^{\mathrm{e}^{-\beta_{\text {asy }}} \mathrm{V}_{\mathrm{c}} \ln \left(\mathrm{V}^{*}(\mathrm{t})\right)} \frac{\mathrm{e}^{-\mathrm{z}}}{\mathrm{z}} \mathrm{dz} \\
& \leq \frac{-1}{\beta_{\text {asy }}} \mathrm{e}^{\frac{\ln \left(\mathrm{V}^{*}(\mathrm{t})\right)}{\left(1-\mathrm{e}^{\left.-\beta_{\text {asy }} \mathrm{t}_{\mathrm{k}}\right)}\right.}}\left(\frac{\left(1-\mathrm{e}^{\left.-\beta_{\text {asy }} \mathrm{t}_{\mathrm{k}}\right)}\right.}{\ln \left(\mathrm{V}^{*}(\mathrm{t})\right) \mathrm{e}^{-\beta_{\text {asy }}} \mathrm{V}_{\mathrm{c}} \ln \left(\mathrm{V}^{*}(\mathrm{t})\right)}\right) \mathrm{e}^{\frac{-\ln \left(\mathrm{V}^{*}(\mathrm{t})\right)}{\left(1-\mathrm{e}^{-\beta_{\text {asy }} \mathrm{t}_{\mathrm{k}}}\right)}} \mathrm{e}^{-\beta_{\text {asy }} \mathrm{V}_{\mathrm{c}} \ln \left(\mathrm{V}^{*}(\mathrm{t})\right)}
\end{aligned}
$$

Now using the fact that $t_{k}<V_{c} \ln V(t)$ when $\beta>0$, we obtain

On the other hand,

$$
\mathrm{I}_{-\beta} \leq \frac{1}{\beta_{\text {asy }}}\left(\frac{\left(1-\mathrm{e}^{-\beta_{\text {asy }} \mathrm{t}_{\mathrm{k}}}\right)}{\ln \left(\mathrm{V}^{*}(\mathrm{t})\right)}\right)
$$

$$
I_{-\beta}=\int_{t_{k}}^{t^{*} m} e^{\ln \left(V^{*}(t)\right) \frac{\left(1-e^{-\beta t}\right)}{\left(1-e^{-\beta t^{*} m}\right.}} d t \geq \int_{t_{k}}^{t^{*} m} \frac{V\left(t^{*}{ }_{m}\right)}{V_{0}} d t \geq V_{k}\left[t^{*}{ }_{m}-t_{k}\right]
$$

Combining (16) and (17) we get,

which implies

$$
\mathrm{V}_{\mathrm{k}}\left[\mathrm{t}^{*}{ }_{\mathrm{m}}-\mathrm{t}_{\mathrm{k}}\right] \leq \frac{1}{\beta_{\text {asy }}}\left(\frac{\left(1-\mathrm{e}^{-\beta_{\text {asy }}} \mathrm{t}_{\mathrm{k}}\right.}{\ln \left(\mathrm{V}^{*}(\mathrm{t})\right)}\right)
$$

$$
\mathrm{t}_{\mathrm{m}}^{*} \leq \mathrm{t}_{\mathrm{k}}+\frac{1}{\mathrm{~V}_{\mathrm{k}} \beta_{\text {asy }}}\left(\frac{\left(1-\mathrm{e}^{-\beta_{\text {asy }} \mathrm{t}_{\mathrm{k}}}\right)}{\ln \left(\mathrm{V}^{*}(\mathrm{t})\right)}\right)
$$

Now we shall estimate $A_{k}$ and $\beta$ using $t^{*}{ }_{m}$. From (13) we get 


$$
A_{k}=\frac{\beta \ln \left(V^{*}(t)\right)}{\left(1-e^{-\beta t^{*}}\right)}=\frac{\beta t^{*}{ }_{m}}{\left(1-e^{-\beta t^{*} \mathrm{~m}}\right)} \frac{\ln \left(V^{*}(t)\right)}{t^{*}{ }_{m}} \geq \frac{\ln \left(V^{*}(t)\right)}{t^{*}{ }_{m}}
$$

Thus the initial growth rate should be at least $\frac{\ln \left(V^{*}(t)\right)}{t^{*} m}$ for $V^{*}(t)>V_{k}$. We also know that $\frac{1}{V_{c}}$ cannot exceed the initial growth rate $A$, when $\beta>0$. Hence for any $\beta$, we have

To estimate $\beta$ from (11) we get

$$
\frac{1}{\mathrm{~V}_{\mathrm{c}}} \leq \mathrm{A} \leq \frac{\varepsilon}{\mathrm{V}_{\mathrm{c}}}, \quad \varepsilon=\frac{\mathrm{V}_{\mathrm{c}} \ln \left(\mathrm{V}^{*}(\mathrm{t})\right)}{\mathrm{t}^{*}{ }_{\mathrm{m}}}>1 .
$$

$$
\frac{t_{k}}{V_{c} \ln \left(V^{*}(t)\right)}=\frac{\beta t_{k}}{\left(1-e^{\left.-\beta t_{k}\right)}\right.} \leq e^{\beta t_{k}}
$$

On the other hand $\frac{t_{k}}{V_{c} \ln \left(V^{*}(t)\right)} \geq \frac{t_{m}}{V_{c} \ln \left(V_{k}\right)}$ for $V^{*}(t)<V_{k}$. Thus we have $\frac{t_{m}}{V_{c} \ln \left(V_{k}\right)} \geq e^{\beta t_{k}}$ which gives

$$
\beta \leq \frac{1}{t_{k}} \ln \left(\frac{t^{*}{ }_{m}}{t_{k}}\right)
$$

Because

$$
\frac{1}{\mathrm{v}_{\mathrm{c}} \ln \mathrm{V}_{\mathrm{k}}}=\frac{1}{\mathrm{t}_{\mathrm{k}}} \text {. }
$$

Remark-1: Using equation (19) we easily calculate the value of $\mathrm{t}_{\mathrm{m}}{ }_{\mathrm{m}}$, by substituting the values of critical growth time $t_{k}$ and corresponding growth rate $\beta$.

Remark-2: The estimated maximum lifespan of tumour will exist theoretically and biologically.

Remark-3: We use the value of the maximum lifespan in experimental, clinical data or tumour therapy at particular time.

\section{Discussion And Conclusion}

Many of the problems in our understanding of the overall growth of tumours arise out of ignorance of the basic characteristics of the proliferating cell population, despite the fact that techniques of investigation of cell population kinetics have been developing rapidly over the past few decades. The application of these techniques to experimental tumours has been shown to be feasible and data are now available on a variety of tumour types. The problem of measuring cell production rate in a tumour is essentially the same as in normal tissues. The slow tumour growth could be the result of a long cell cycle time. As regards cell proliferation in all types of tumours, the experimental difficulties are very great. However, in the light of a detailed knowledge of the situation in experimental tumours it is possible to plan simple investigation.

Most of the information about tumour growth rates comes from studies performed long ago and not known clearly the maximum volume size of individual tumours and groups of tumours. The expectation that tumour growth under ideal conditions would prove to be exponential until it terminates with the exhaustion of the host has not been borne out in many careful studies of the growth of a wide variety of tumours. The specific growth rate of tumours is usually not constant even for a short time, but decreases steadily. So, the present study we have shown that tumour growth is well described by a Gompertz function, according to which the times required to double the tumour volume (VRD)increase according to an exponential function. The Gompertzian model is a classical continuous model useful in describing population dynamics; in particular, it is a very efficient mathematical model to describe tumour growth in humans and animals. Especially in experimental oncology, the Gompertzian model is most widely used to describe in vivo tumour growth. Qualitatively, this model gives exponential growth at early time periods which then saturates at later time periods (decelerating growth).

Sensitivity analysis can be used to determine the functional relationship between tumour size or growth rate and the constituent rates (e.g., fecundity, survival, growth, maturation, recruitment, movement), and to project changes in tumour growth rate and size as vital rates change. This will give the result

It shows that the value of parameter $\frac{\partial \beta}{\partial \mathrm{V}^{*}(\mathrm{t})} \geq 0, \frac{\partial \beta}{\partial \mathrm{V}_{\mathrm{c}}} \leq 0$ and $\frac{\partial \beta}{\partial \mathrm{t}_{\mathrm{m}}} \leq 0$.

$B$ is increases when value of $V^{*}(t)$ is increases, $\beta$ is decrease when the value of $V_{c}$ increase and also $\beta$ is decrease when the value of $t_{m}$ increases. The parameter $\beta$ will be more sensitive towards the cumulative volume $V_{c}$ and maximum lifespan tm. In the behaviour of $\beta$, it does not change often or accordingly the change of volume of tumour, since the ratio between initial mitosis rate $A$ and growth deceleration factor $\beta$ is constant. The disruption of cell cycle or alterations of normal cells properties will 
leads to the formation of an initial tumour, from that we get $\mathrm{A}$ and this is not depends on neither the number of cells to be formed nor make changes in the tumour growth.

Also observed that to have a unique independent parameter $\mathrm{A}$; it is necessary that

$\frac{1}{V_{c}} \leq A$. This data is not dependent on the value of $V^{*}(t)$, so it is not affected by any large number of solid tumour cells. So we calculate the unique $\beta$ by Gompertz tumour growth model, even the specific volume data of solid tumour cells were not given. Hence, we can estimate the growth deceleration parameter using the equation (6), in the absence of specific volume data of large number solid tumour cells at particular time, also the value of $A$ can be calculated through the unique value of $\beta$.

An exact mathematical description of our model of tumour cell proliferation is given by a Gompertz model with the assumption that there is no upper bound on maximum lifespan. Hence, we made use of Gompertzian growth with an infinite time $[t \in(0, \infty)]]$. Clearly, this is not correct. But we do not know what is the maximum lifespan? and we do know that at least one must exist. Observe that individual cell lifespans may vary, but they are bounded by the value of $t^{*}$.

The biological impact of such a restriction is discussed in Liu and Witten [23]. Given the assumption of a maximal lifespan $\mathrm{t}^{*} \mathrm{~m}$, we must adjust our Gompertz tumour growth model to take this into account.

An asymptotic solution is useful in the study of qualitative behaviour of solution. The asymptotic solution of the basic equation(6) for a large $V^{*}(t)$ is given, when $\frac{t_{k}}{V_{c} \ln \left(V^{*}(t)\right)}<1$ and $\frac{t_{k}}{V_{c} \ln \left(V^{*}(t)\right)}=$ 1.

We can estimate the value of critical time $t_{k}$, critical volume $V_{k}$ by the method is given in [31] then substitute in equation (19) to get the maximum lifespan $\mathrm{t}_{\mathrm{m}}{ }_{\mathrm{m}}$. This is useful when do the experiments and clinical study or test. Also it may apply in the theoretical predictions for the tumour therapy and treatments. The value of $\beta$ is a continuous function in the variables $\frac{1}{V_{c}} ; t_{m}$ and $V^{*}(t)$ from initial growth time to critical growth time. We calculated the maximum life span $\left(\mathrm{t}_{\mathrm{m}}^{*}\right)$ from our obtained equation (19). From table-I,we observe that our derivation is better for all tumour maximum life span $\mathrm{t}_{\mathrm{m}}^{*}$. In table-I we compared the values of $t_{m}, t_{k}$ and $t^{*}{ }_{m}$. All the critical time values are less than the maximum life span $\mathrm{t}^{*}{ }_{\mathrm{m}}$.

In table -I the Rat tumour of type Walker( W26b1) and Walker (W12a7) the time of death $t_{m}$ are more than the $\mathrm{t}_{\mathrm{m}}^{*}$, since these two tumour volume of cells are very small and its theoretical upper limit is very high. The remaining data fit well with other experimental data. The large volume of tumour cells are fit well with the asymptotic solution and other characteristics of Gompertz tumour growth model. The basic data and other calculations of parameters are available in [2] and [31].

The purpose of this discussion is to estimate the maximum lifespan of Gompertz tumour growth parameter at particular time. Such a method is necessary when attempting to estimate the growth rate in a Gompertz tumour growth model, and its maximum lifespan.

From these analyses, we believe that our model and methods will provide a useful approach to prediction of experimental and clinical tumour growth.

Table-I Analysis of theoretical Gompertz functions in terms of $t_{m}, t_{k}$ and $t^{*}{ }_{m}$ which depends on the tumour

\begin{tabular}{|c|c|c|c|c|c|}
\hline S.No & tumour type & $\beta$ & $\mathrm{t}_{\mathrm{m}} /$ days & $\mathrm{t}_{\mathrm{k}} /$ days & $\mathrm{t}_{\mathrm{m}}^{*} /$ days \\
\hline & Mouse: & & & & \\
\hline 1. & Krebs & 0.411 & - & 10.4429 & 763.54 \\
\hline 2. & Ehrlich & 0.009 & - & 331.1687 & 6523.36 \\
\hline 3 & 6C3HED,high dose & 0.012 & 425.9917 & 170.2028 & 1312.15 \\
\hline 4 & 6C3HED,low dose & 0.0116 & 567.3520 & 141.5309 & 730.899 \\
\hline 5 & EO771 & 0.063 & 79.4302 & 32.9247 & 262.035 \\
\hline \multirow[t]{2}{*}{6} & Osteosarcomas & 0.159 & 38.9177 & 18.2688 & 333.60 \\
\hline & Rat: & & & & \\
\hline 7 & Walker,W26b1 & 0.0218 & 341.2281 & 42.3284 & 106.51 \\
\hline 8 & Walker,W12a7 & 0.0205 & 342.2281 & 51.0880 & 145.60 \\
\hline 9 & Walker,W10a6 & 0.039 & 59.1180 & 36.7349 & 153.91 \\
\hline 10 & Walker,W10b4 & 0.003 & - & 79.8275 & 101.43 \\
\hline 11 & R39Sarcoma,R3a7 & 0.124 & 42.4407 & 28.4854 & 974.17 \\
\hline 12 & 7R39Sarcoma,R4c4 & 0.078 & - & 32.2799 & 400.33 \\
\hline 13 & R39Sarcoma,a7R3 & 0.063 & - & 62.7635 & 3273.04 \\
\hline \multirow[t]{2}{*}{14} & Flexne-Jobling & $0.049-$ & - & 43.9302 & 378.11 \\
\hline & Rabbit: & & & & \\
\hline 15 & Brown-Pearce & 0.0169 & 45.7709 & 28.9986 & 47.6088 \\
\hline
\end{tabular}

The source of data for each species is given in $[2,31]$. 


\section{Acknowledgement}

The work by M.Pitchaimani is supported by the SERC-DST (Ministry of Science and Technology, Government of India) Reference number:SR/S4/MS-677/10.

\section{References}

[1]. Al-Dweri,F.M.O., Guirado,D., Lallena,A. M., and Pedraza,V. E_ect on tumour control of time interval between surgery and postoperative radiotherapy: an empirical approach using Monte Carlo simulation, Phys. Med. Biol., Vol.49, p.2827-2839, (2004).

[2]. Anna Kane Laird. Dynamics of Tumour Growth, Br. J. Cancer., Vol.18(3), p.490-502, (September 1964).

[3]. Anna Kane Laird. Dynamics of Tumour Growth: Comparisons of growth rates and extrapolation of growth curve to one cell, Br J Cancer., Vol. 19, p.278-291, (1965).

[4]. Araujo,R.P., and McElwain,L.S. A History of the Study of Solid Tumor Growth: The Contribution of Mathematical Modeling, Bulletin of Mathematical Biology, Vol.66, p.1039-1091, (2004).

[5]. Bass,L., Green,H.S., and Boxenbaum,H. Gompertzian mortality derivedfrom competitionbetween cell-types: congenial, toxicologic and biometric determinants of longevity, J.Theor.Biol., Vol.140, p.263-278, (1989).

[6]. Bassukas,I.D. Comparative Gompertzian analysis of alterations of tumour growth patterns, Cancer Research, Vol.54, p.4385-4392, (1994).

[7]. Boag,J.W. Maximum likelihood estimates of the proportion of patients cured by cancer therapy, J.R.Stat. Soc., Vol.11, p.15-53, (1949).

[8]. Byrne,H.M., and Chaplain,M.A. Growth of necrotic tumours in the presence and absence of inhibitors, Math.Biosci., Vol.135, p.187-216, (1996).

[9]. Byrne,H.M. A weakly nonlinear analysis of a model of avascular solid tumour growth, J.Math.Biol., Vol.39, p.59-89, (1999).

[10]. Collins,V.P., Loe e,R. K., and Tivery,H. Observations on growth rates of human tumors, Am.J. Roentgenol Radium The.r Nucl. Med., Vol.76(5), p.988-1000, (1956).

[11]. Ferrante,L., Bompadre,S., Possati,L., and Leone,L. Parameter estimation in a Gompertzian stochastic model for tumor growth, Biometrics, Vol.56, p.1076-1081, (2000).

[12]. Finch,C.E., Pike,M.C., and Witten,M. Slow mortality rate accelerations during ageing in some animals approximate that of humans, Science, Vol.249, p.902-905, (1990)

[13]. Folkman,J. Tumour angiogenesis, Adv.Cancer.Res., Vol.19, p.331-358, (1974).

[14]. Folkman,J. The vascularisation of tumours, In:Cancer Biology, (edited by E.C.Friedberg), Scienti_c American, p.115-124, (1976).

[15]. Folkman,J., and Hochberg,M. Self-regulation of growth in three dimensions, J.Exp.Med., Vol.138(4), p.745-753,(1973).

[16]. Fuchshuber,P., Gunther,M., Feaux de Lacroix,W., and Fischer,R. A Mathematical model for metastatic growth illustrated by in vivo and in vitro growth of a transplantable mammary carcinoma in mice, Anticancer Research, Vol.6, p.819-828, (1986)

[17]. Gompertz,B. On the nature of the function expressive of the law of human mortality, and on a new mode of determining the value of Life Contingencies, Trans.R.Philos. Soc., Vol.115, p.513-585, (1825).

[18]. Gradshteyn,I.S., and Ryzhik,I.M. Table of Integrals, Series and Products, Academic Press, (1980).

[19]. Iarosz,K.C., Martins,C.C., Batista,A.M., Viana,R.L, Lopes,S. R, Caldas,I.L., and Penna,T.J.P. On a cellular automaton with time delay for modelling cancer tumours, Journal of Physics, Conference Series, Vol.285, p.1-10, (2011).

[20]. Kerr,J.F.R. Shrinkage necrosis; a distinct mode of cellular death, J.Path., Vol.105, p.13-20, (1971).

[21]. Lakshminarayanan,E.S., and Pitchaimani,M. Existence of Gompertz parameters and its asymptotic formulae for a large population, Appl. Math. Lett., Vol.17(2), p.173-180, (2004).

[22]. Lakshminarayanan,E.S., and Pitchaimani,M. Existence of critical Gompertz parameters and its asymptotic expression, Comp.Math. with applications, Vol.55, p.1302-1309, (2008)

[23]. Liu,X., and Witten,.M. A biologically based explanation for mortality crossover in human populations, Gerontologist, Vol.35, p.609-615, (1995).

[24]. Maggelakis,S.A., and Adam,J.A. Mathematical model of prevascular growth of a spherical carcinoma, Math.Comput.Modelling., Vol.13, p.23-38, (1990).

[25]. McCredie,J.A., Inch,W.R., Kruuv,J., and Watson,T.A. The rate of tumour growth in animals, Growth, Vol.29, p.331-347, (1965).

[26]. McElwain,D.L.S., and Morris,L.E. Apoptosis as a volume loss mechanism in mathematical models of solid tumour growth, Math.Biosci., Vol.39, p.147-157,(1978).

[27]. Michelson,S., and Leith,J. Positive feedback and angiogenesis in tumour growth control, Bull.Math.Biol., Vol.59, p.233-254, (1997).

[28]. Muthukarrupan,V.R., Kubai,L., and Auerbach,R. Tumour-induced neovascularisation in the mouse eye, J.Natl.Cancer Inst., Vol.69, p.699-705, (1982).

[29]. Norton,L., Simson,R., Brereton,H.D., and Bogden,A.E. Predicting the course of Gompertizian Growth, Nature, Vol.264, p.542-545, (1976).

[30]. Panetta,J.C. A Mathematical model of periodically pulsed chemotherapy: Tumour recurrence and metastasis in a competitive environment, Bull.Math.Biol., Vol.58, p.425-447, (1996)

[31]. Pitchaimani,M., and Somasundara Ori,G. Existence of Critical Gompertz Parameter for Solid Tumour Growth Model and its Asymptotic Expression, International Journal of Emerging Science and Engineering, Vol.2(5), p.36-41, (2014).

[32]. Pitchaimani,M., and Somasundara Ori,G. Stability Analysis of Gompertz tumour growth model Parameters, Journal of Advances in Mathematics (Council of Innovative Research), Vol.7(3), p.1293-1304, (2014).

[33]. Qi,A.S., Zheng,X., Du,C.Y., and An,B.S. A cellular automaton model of cancerous growth, J.theor.Biol., Vol.161, p.1-12, (1993).

[34]. Rygaard,K., and Spang-Thomsen,M. Quantitation and Gompertzian analysis of tumour growth, Breast Cancer: Res.Treat., Vol.46(2-3), p.303-312, (1997).

[35]. Steel,G.G., and Lamerton,L.F. The Growth rate of Human tumours, Br.J.Cancer., Vol.20(1), p.74-86,(1966),

[36]. Sutherland,R.M. Cell and environment interactions in tumour micro regions: The multicell spheroid model, Science, Vol.240, p.177-184, (1988)

[37]. Sutherland,R.M., and Durand,R.E. Growth and cellular characteristics of multi cell spheroids, Recent Results in Cancer Research, Vol.95, p.24-49, (1984)

[38]. Tyurin,N.Y., Yakovlev,A.Y., Shi,J., and Bass,L. Testing a model of aging in animal experiments, Biometrics, Vol.51(1), p.363-372, (1995).

[39]. Xu,X. The biological foundation of the Gompertz model,International Journal of Biomedical Computing, Vol.20, p.35-39, (1987). 